\title{
Order statistic s from Beta inverse Weibull distribution and characterizations \\ Dr/Abeer S.Mohamed
}

Department of Statistics, Faculty of Commerce (Girls' Branch), Al-Azhar University, Cairo, Egypt

الملخص

يعتبر توزيع معكوس و اييل من اكثر التوزيعات الاحتمالية استخداما في دراسة نظريات الصلاحية و التي تستخدم بكثره في العلوم التطبيقيه و والفيزيائيه وبداية التئية

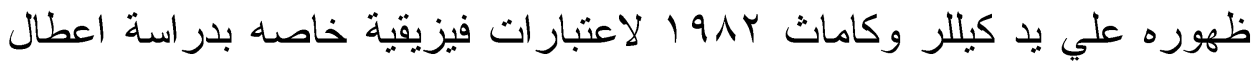

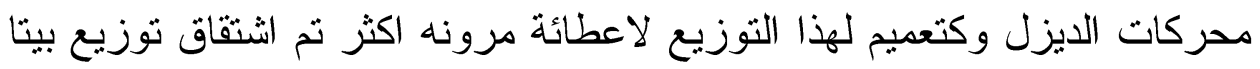

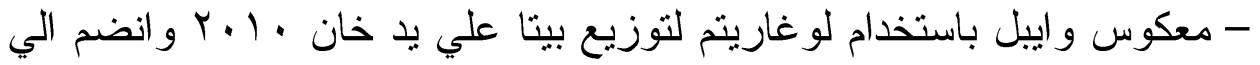

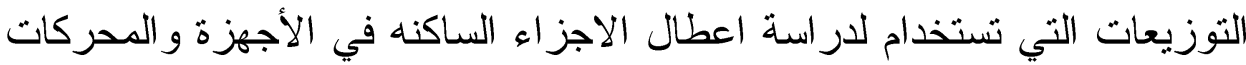

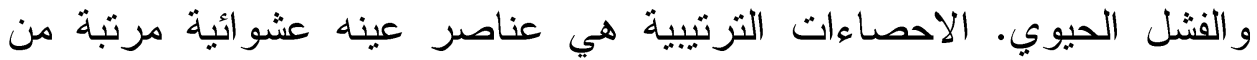

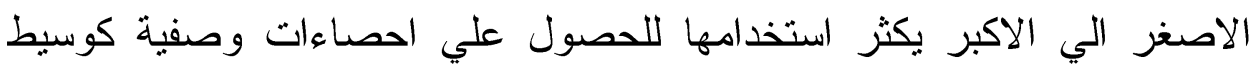

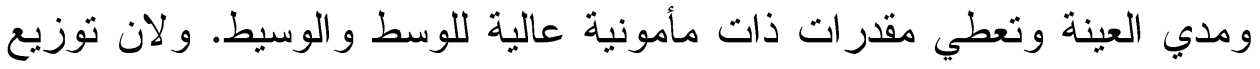

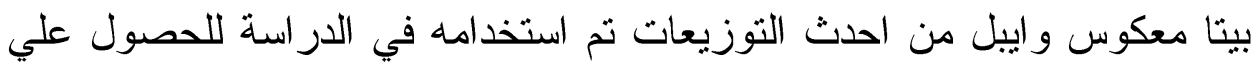

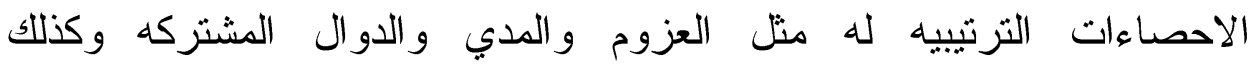
الاحصاءات التزتيبه للتوزيع عند قطعه بشكل مزدوج (الطرف الآيهن والهاء الايسر). 


\section{Abstract}

Inverse Weibull distribution (IW) referred to as the Beta Inverse Weibull distribution( BIW) is generated from the logit of a beta random variable to be considered as a new reliability model. The basic distribution theory of order statistics (ordered random variables) is developed for both finite and asymptotic random samples.Order statistics provide simple robust estimators of location such as the median or trimmed means. The smallest, largest,or middle observations for a given random sample can be computed using order statistic.

In this paper the order statistics from Beta inverse (BIW) distribution is studied. The cumulative distribution and the density for maximum value and minimum are also presented, the exact expression for the single and double moments of order statistics from BIW distribution are derived, finally the order statistic for doubly truncated of the BIW, its $r^{\text {th }}$ single and joint $\mathrm{r}^{\text {th }}$ moments are presented.

Keywords: Beta inverse Weibull, order statistics, single moment of order statistic, double moment of order statistic, doubly truncated. 
Order statistics from Beta inverse Weibull distribution

$\sim$ Abeer S.Mohamed

\section{Research Methodology}

1.Selection of the research topic: distribution beta inverse Weibull distribution has been chosen as a modern distribution to study its properties using order statistics has not been studied before.

2.The importance of research: the most continues distributions related to that used in the distributions of life for estimating the reliability and failure in the industrial, medical and engineering field distribution are Weibull and inverse Weibull. beta inverse Weibll distribution has been derived recently to give the prior distributions more flexibility in the presentation of the data to the medical, engineering and industrial study.

3 .The research plan: research plan adopted to study the references that were used order statistics with continuous distributions. Then we using the theorems of the order statistic to put the characteristic to the new distribution beta inverse Weibull

4.Search Results: we drive the cumulative probability density function and probability density function to the greatest value and the lowest value of the distribution. the exact expression for the single and double moments of order statistics from BIW distribution are derived, finally the order statistic for doubly truncated of the BIW was presented. 
Order statistics from Beta inverse Weibull distribution

$\sim$ Abeer S.Mohamed

\section{Introduction}

Order statistics has been used in many real-life applications involving data relating to life testing studies and the BIW distribution was introduced by Khan (2010) as a new reliability model which is generated from the logit of a beta random variable. This model has been introduced as a life time distribution giving more flexibility than the inverse Weibull distribution which was introduced by Keller and Kamath (1982) and was based on the IW distribution. Many authors have studied order statistics and the associated inference for many distributions. Shawky and Bakoban (2009) derived the order statistics from an exponentiated gamma distribution with exact expression for the single and double moments. Raqab and Ahsanullah (2001) obtained the BLUE's of the location and scale parameters of generalized exponential distribution based on order statistics. Balakrishnan and Chan (1998) have discussed linear estimation for log-gamma parameters based on order statistics.

Mahmoud et al. ( 2003 ) introduced the exact expression for the single and double moments of order statistics from the inverse Weibull distribution and calculated the variances and covariance's and they used these moments to obtain the BLUE's of the location and scale parameters of inverse Weibull distribution under Type-II censored sample. Mohamed (2013) introduced the Bayesian estimation to obtain the estimators of the scale parameter $\alpha$ for BIW distribution. The Bayes estimators are derived by considering non-informative and informative prior distributions based on LINEX, general 
Order statistics from Beta inverse Weibull distribution

$\sim$ Abeer S.Mohamed

entropy and binary loss functions. Mohamed (2014) presented a comparison between Bayesian estimation and maximum likelihood estimation for BIW.

Aboutahoun and Al-Otaibi (2009) are derive the recurrence relations between the single and the product moments for order statistics from doubly truncated Makeham distribution.

Let $Y_{1}, Y_{2}, \ldots, Y_{n}$ be a random sample of size $\mathrm{n}$ from BIW distribution with the probability density function (p.d.f) is given by

$$
f(y)=\frac{\beta \alpha^{-\beta}}{\beta(\mathrm{a}, \mathrm{b})} \mathrm{y}^{-(\beta+1)} \mathrm{e}^{-\mathrm{a}(\alpha \mathrm{y})^{-\beta}}\left[1-\mathrm{e}^{-(\alpha \mathrm{y})^{-\beta}}\right]^{\mathrm{b}-1}
$$

for $y \geq 0, \alpha>0, \beta>0, a>0$ and $b>0$.

and the cumulative distribution function is given by 1.2.

$F(y)=$

$\frac{\Gamma(b)}{\beta(a, b)} \sum_{i=0}^{\infty} \frac{(-1)^{i}}{\Gamma(b-i) i !(a+i)} e^{-(a+i)(\alpha y)^{-\beta}}$

This paper is organized as it follows. Section 2 introduces the order statistic, the minimum, the maximum of density and the cumulative density function of BIW. We derive exact expression for the single moments of order distribution to the BIW in section 3.

\section{The Order statistic of BIW}

Let $Y_{1: n} \leq Y_{2: n} \leq \ldots \leq Y_{n: n}$ denote the order statistics for the sample of size $\mathrm{n}$ from the BIW distribution and $Y_{j: n,}, j=1,2, \ldots, n$ is the $\mathrm{j}^{\text {th }}$ order statistic with probability density function given by David (1981). 


$$
f_{j: n}(y)=\mathrm{C}_{\mathrm{j}: n} f(y)[F(y)]^{j-1}[1-F(y)]^{n-j} . \quad-\infty<y<\infty
$$

where $f($.$) and F($.$) are given in (1.1) and (1.2), respectively,$ and

$$
\mathrm{C}_{\mathrm{j}: \mathrm{n}}=\frac{n !}{(\mathrm{n}-\mathrm{j}) !(\mathrm{j}-1) !} \text {, }
$$

And the joint p.d.f of $Y_{j: n}$ and $U_{k: n}$ with integer $\mathrm{j}$ and $\mathrm{k}$ $1 \leq j \leq k \leq n$ is given by David (1981) as follows:

$$
f_{j ; k: n}(y, u)=\mathrm{C}_{\mathrm{j}, \mathrm{k}: \mathrm{n}}(\mathrm{F}(\mathrm{u}))^{\mathrm{j}-1}(\mathrm{~F}(\mathrm{y})-\mathrm{F}(\mathrm{u}))^{\mathrm{k}-\mathrm{j}-1}(1-\mathrm{F}(\mathrm{y}))^{\mathrm{n}-\mathrm{k}} f(y) f(u)
$$

where $C_{j, k: n}=\frac{n !}{(j-1) !(k-j-1) !(n-k) !}$

and $\mathrm{f}($.$) and \mathrm{F}($.$) are given in (1.1) and (1.2), respectively$

\subsection{The Cumulative distribution and the density for the} smallest order statistic

\section{Theorem:}

For $Y_{1: n} \leq Y_{2: n} \leq \ldots \leq Y_{n: n} \mathrm{i}$ id continuous random sample of size $\mathrm{n}$ with p.d.f and c.d.f with continuous distribution then the smallest order statistic is given as:

$$
\begin{aligned}
\mathrm{f}_{(I)}(y) & \left.=\frac{\mathrm{d}}{\mathrm{dy}}\left(1-(1-\mathrm{F}(\mathrm{y}))^{\mathrm{n}}\right)=\mathrm{n} 1-\mathrm{F}(\mathrm{y})\right)^{\mathrm{n}-1}=\mathrm{n}(1-\mathrm{F}(\mathrm{y}))^{\mathrm{n}-1} \frac{\mathrm{d}}{\mathrm{dy}} \mathrm{F}(\mathrm{y}) \\
& =\mathrm{nf}(\mathrm{y})(1-\mathrm{F}(\mathrm{y}))^{\mathrm{n}-1}
\end{aligned}
$$

$$
\begin{aligned}
& \text { And } \\
& \begin{array}{l}
\mathrm{F}_{(1)}(\mathrm{y})=\mathrm{P}\left(\mathrm{Y}_{(1)}<\mathrm{y}\right)=1-\mathrm{P}\left(\mathrm{Y}_{(1)}>\mathrm{y}\right)=1-\mathrm{P}\left(\mathrm{Y}_{1}>\mathrm{y} \ldots \mathrm{P}\left(\mathrm{Y}_{\mathrm{n}}>\mathrm{y}\right)\right. \\
\quad=1-(1-\mathrm{F}(\mathrm{y}))^{\mathrm{n}}
\end{array}
\end{aligned}
$$


Order statistics from Beta inverse Weibull distribution

$\sim$ Abeer S.Mohamed

From (2.5) and (2.6) and using (1.1) and (1.2) (for BIW distribution), then

$$
\begin{aligned}
& , f_{(1)}(y)=M y^{-(\beta+1)} e^{-a(\alpha y)^{-\beta}}\left[1-e^{-(\alpha y)^{-\beta}}\right]^{b-1} \\
& \quad *\left(1-\frac{\Gamma(b)}{\beta(a, b)} \sum_{i=0}^{\infty} \frac{(-1)^{i}}{\Gamma(b-i) i !(a+i)} e^{-(a+i)(\alpha y)^{-\beta}}\right)^{\mathrm{n}-1}
\end{aligned}
$$

Where $M=n \frac{\beta \alpha^{-\beta}}{\beta(a, b)}$

and

$\mathrm{F}_{(1)}(\mathrm{y})=1-\left(1-\frac{\Gamma(b)}{\beta(a, b)} \sum_{i=0}^{\infty} \frac{(-1)^{i}}{\Gamma(b-i) i !(a+i)} e^{-(a+i)(\alpha y)^{-\beta}}\right)^{\mathrm{n}}$

2.2 The Cumulative distribution and the density the largest order statistics:

\section{Theorem:}

If $Y_{1}, Y_{2}, \ldots, Y_{n}$ be a random sample of size $n$ from a population with continuous p.d.f $f(y)$, then the p.d.f. and c.d.f of the largest order statistics $Y_{(n)}$ is given as:

$$
\begin{aligned}
& f_{(n)}(y)=\frac{\mathrm{d}}{\mathrm{dy}} \mathrm{F}(\mathrm{y})^{\mathrm{n}}=\mathrm{n} \mathrm{F}(\mathrm{y}){ }^{\mathrm{n}-1} \frac{\mathrm{d}}{\mathrm{dy}} \mathrm{F}(\mathrm{y}) \\
& =\mathrm{nf}(\mathrm{y}) \mathrm{F}(\mathrm{y})^{\mathrm{n}-1}
\end{aligned}
$$

\section{And}

$$
\begin{aligned}
\mathrm{F}_{(\mathrm{n})}(\mathrm{y}) & =\mathrm{P}\left(\mathrm{Y}_{(\mathrm{n})}<\mathrm{y}\right)=1-\mathrm{P}\left(\mathrm{Y}_{(\mathrm{n})}>\mathrm{y}\right)=\mathrm{P}\left(\mathrm{Y}_{1}<\mathrm{y} \ldots \mathrm{P}\left(\mathrm{Y}_{\mathrm{n}}<\mathrm{y}\right)\right. \\
& =\mathrm{F}(\mathrm{y})^{\mathrm{n}},
\end{aligned}
$$

Then., for the BIW in (1.1) and

$$
\begin{gathered}
f_{(n)}(y)=\mathrm{M} y^{-(\beta+1)} e^{-a(\alpha y)^{-\beta}}\left[1-e^{-(\alpha y)^{-\beta}}\right]^{b-1} \\
*\left(\frac{\Gamma(b)}{\beta(a, b)} \sum_{i=0}^{\infty} \frac{(-1)^{i}}{\Gamma(b-i) i !(a+i)} e^{-(a+i)(\alpha y)^{-\beta}}\right)^{\mathrm{n}-1}
\end{gathered}
$$


Order statistics from Beta inverse Weibull distribution

$\sim$ Abeer S.Mohamed

\section{and}

$\mathrm{F}_{(\mathrm{n})}(\mathrm{y})=$

$$
\left(\frac{\Gamma(b)}{\beta(a, b)} \sum_{i=0}^{\infty} \frac{(-1)^{i}}{\Gamma(b-i) j !(a+i)} e^{-(a+i)(\alpha y)^{-\beta}}\right)^{\mathrm{n}}
$$

(2.12)

\section{The moments}

The $\mathrm{s}^{\text {th }}$ moments of the $\mathrm{j}^{\text {th }}$ order statistics from BIW is given by

$$
\mu_{j: n}^{(s)}=E\left(y_{j: n}^{s}\right)=C_{j: n} \int_{-\infty}^{\infty} y^{s} f(y)(F(y))^{j-1}(1-F(y))^{n-j} d y
$$

Using the equations (1.1), (1.2) and (3.1), then the single moment of order statistics from the BIW distribution can be written as follows:

$$
\begin{aligned}
& \mu_{j: n}^{(s)}=E\left(y_{j: n}^{s}\right)=C_{j: n} A \int_{-\infty}^{\infty} \mathrm{y}^{-(\beta+1-s)} \mathrm{e}^{-a(\alpha y)^{\beta \beta}}\left(1-\mathrm{e}^{-(\alpha y)^{\beta}}\right)^{b-1} \\
& \left.\left.\left(\sum_{i=0}^{\infty} \frac{(-1)^{i}}{\Gamma(a-i) i(a+i)}\right) e^{-(a+i)(\alpha y)^{-\beta}}\right)^{j-1}\left(1-\frac{\Gamma b}{\beta(a, b)} \sum_{i=0}^{\infty} \frac{(-1)^{i}}{\Gamma(a-i) i(a+i)}\right) e^{-(a+i)(\alpha y)^{-\beta}}\right)^{n-j} d y
\end{aligned}
$$

\section{Where}

$$
A=\left(\frac{\Gamma b}{\beta(a, b)}\right)^{i}
$$

The equation (3.2) can be solved numerically

The double (h,s)th moment of $Y_{j: n}$ and $U_{k: n}, 1 \leq j \leq k \leq n$ is given by

$$
\begin{gathered}
\mu_{j, k: n}^{(h, s)}=E\left(y_{j: n}^{s} u_{k: n}^{h}\right)=C_{j, k: n} \int_{-\infty}^{\infty} \int_{u}^{\infty} y^{s} u^{h} f(y) f(u)(F(y))^{j-1}(1-F(y))^{n-k} \\
(F(y)-F(u))^{j-k-1} d y d u
\end{gathered}
$$


Order statistics from Beta inverse Weibull distribution

$\sim$ Abeer S.Mohamed

where $f($.$) and F($.$) are given in (1.1) and (1.2), respectively$ and $C_{j ; k: n}$ is given in (2.4)

if we would like to derive the c.d.f of the sample range $R_{n}$ of a random sample of size $\mathrm{n}$ form a BIW distribution,

\section{Theorem :}

Let $R_{n}:=Y_{n: n}-Y_{1: n}$ be the range of a random sample of size $\mathrm{n}$ from a continuous distribution with $(-\infty, \infty)$. Then, for any real number $r>0$, we have

$$
F\left(R_{n}\right)=n \int_{-\infty}^{\infty}(F(y+r)-F(y))^{n-1} f(y) d y
$$

Using (1.1) and (1.2) for BIW,

$$
\begin{aligned}
& F\left(R_{n}\right)=\int_{-\infty}^{\infty} \frac{\beta \alpha^{-\beta}}{\beta(a, b)} \mathrm{y}^{-(\beta+1)} \mathrm{e}^{-\mathrm{a}(\alpha y)^{-\beta}}\left(1-\mathrm{e}^{-(\alpha \mathrm{y})^{-\beta}}\right)^{\mathrm{b}-1} \\
& \left.\left(I-\frac{\Gamma b}{\beta(a, b)} \sum_{i=0}^{\infty} \frac{(-1)^{i}}{\Gamma(a-i) i(a+i)}\right) e^{-(a+i)(\alpha y)^{-\beta}}\right)^{n-1} d y
\end{aligned}
$$

where

$$
I=F(y+r)=\frac{\Gamma(b)}{\beta(a, b)} \sum_{i=0}^{\infty} \frac{(-1)^{i}}{\Gamma(b-i) i !(a+i)} e^{-(a+i)(\alpha(y+r))^{-\beta}}
$$

\subsection{Doubly truncated}

We can derive The doubly truncated p.d.f of continuous random variable from the BIW from (1.1) as follows:

$$
g(y)=\frac{f(y)}{P-Q}=\frac{\beta \alpha^{-\beta}}{P-O(\beta(a, b))} \mathrm{y}^{-(\beta+1)} \mathrm{e}^{-\mathrm{a}(\alpha y)^{-\beta}}\left(1-\mathrm{e}^{-(\alpha y)^{-\beta}}\right)^{\mathrm{b}-1}, Q_{1} \leq y \leq P_{1}
$$

\section{Where}

$$
\left.P=\frac{\Gamma b}{\beta(a, b)} \sum_{i=0}^{\infty} \frac{(-1)^{i}}{\Gamma(a-i) i(a+i)}\right) e^{-(a+i)\left(\alpha P_{1}\right)^{-\beta}}
$$


Order statistics from Beta inverse Weibull distribution

$\sim$ Abeer S.Mohamed

$$
\left.Q=\frac{\Gamma b}{\beta(a, b)} \sum_{i=0}^{\infty} \frac{(-1)^{i}}{\Gamma(a-i) i \cdot(a+i)}\right) e^{-(a+i)\left(\alpha Q_{1}\right)^{-\beta}}
$$

and $\mathrm{Q}, \mathrm{P}(0<\mathrm{Q}<\mathrm{P}<1)$ are respectively the proportions of the truncation on the left and the right of BIW distribution in (1.1),

where the doubly truncated BIW can be used in some situation that depend on the time start $t_{0} \neq 0$

The cumulative distribution function c,d.f of the doubly truncated BIW is given by

$G(x)=Q_{2}-\left[\frac{\beta \alpha^{-\beta}}{P-O(\beta(a, b))} \mathrm{y}^{-(\beta+1)} \mathrm{e}^{-\mathrm{a}(\alpha \mathrm{y})^{-\beta}}\left(1-\mathrm{e}^{-(\alpha \mathrm{y})^{-\beta}}\right)^{\mathrm{b}-1}\right]$

where $Q_{2}=\frac{Q}{P-Q}$

(3.10)

Let $Y_{1: n} \leq Y_{2: n} \leq \ldots \leq Y_{n: n}$ is a sample of order statistics of size $\mathrm{n}$ from the doubly truncated BIW distribution which given in (3.7) and the c.d.f in (3.10) then we can derive the p.d.f for the $\mathrm{j}^{\text {th }}$ order statistic as follows:

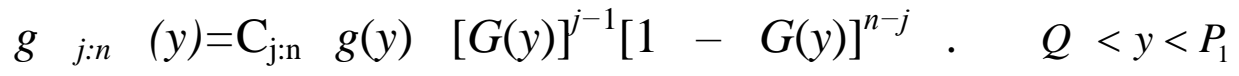
(3.11)

where $\mathrm{g}($.$) and \mathrm{G}($.$) are given in (3.7) and (3.10), respectively,$ and

$$
\mathrm{C}_{\mathrm{j}: \mathrm{n}}=\frac{n !}{(n-\mathrm{j}) !(\mathrm{j}-1) !},
$$


Order statistics from Beta inverse Weibull distribution

$\sim$ Abeer S.Mohamed

And the joint p.d.f of $Y_{j: n}$ and $U_{k: n}$ with integer $\mathrm{j}$ and $\mathrm{k}$ $1 \leq j \leq k \leq n$ is given by David (1981) as follows:

$g_{j ; k: n}(y, u)=\mathrm{C}_{\mathrm{j} ; \mathrm{k}: \mathrm{n}}(\mathrm{G}(\mathrm{u}))^{\mathrm{j}-1}(\mathrm{G}(\mathrm{y})-\mathrm{G}(\mathrm{u}))^{\mathrm{k}-\mathrm{j}-1}(1-\mathrm{G}(\mathrm{y}))^{\mathrm{n}-\mathrm{k}} g(y) g(u)$

(3.12)

$$
C_{j ; k: n}=\frac{n !}{(j-1) !(k-j-1) !(n-k) !}
$$

and $\mathrm{g}($.$) and \mathrm{G}($.$) are given in (3.7) and (3.10), respectively.$

The $s^{\text {th }}$ moments of the $j^{\text {th }}$ order statistics from BIW(the single moments) is given by

$$
\mu_{j: n}^{(s)}=E\left(y_{j: n}^{s}\right)=C_{j: n} \int_{-Q_{1}}^{P_{1}} y^{s} \mathrm{~g}(y)(G(y))^{j-1}(1-G(y))^{n-j} d y, 1 \leq r \leq n
$$

The equation (3.14) can be solved numerically

The double $(\mathrm{h}, \mathrm{s})^{\text {th }}$ moment of $Y_{j: n}$ and $U_{k: n}, 1 \leq j \leq k \leq n$ is given by

$$
\begin{gathered}
\mu_{j, k: n}^{(h, s)}=E\left(y_{j: n}^{s} u_{k: n}^{h}\right)=C_{j, k: n} \int_{0}^{\infty} \int_{y}^{\infty} y^{s} u^{h} g(y) g(u)(G(y))^{j-1}(1-G(y))^{n-k} \\
(G(y)-G(u))^{j-k-1} d y d u
\end{gathered}
$$

\subsection{Special Cases}

The single and double moments of order statistic to the many of distributions can be derived from (3.2) and (3.4) as a special cases from BIW.

for $\mathrm{a}=1, \mathrm{~b}=1$, and $\beta=2$ the distribution in (1.1) reduces to standard inverse Rayleigh distribution (SIR), i.e.,

$$
f(y)=\frac{2}{\alpha^{2} y^{3}} e^{-\frac{1}{(\alpha y)^{2}}}, \quad \text { for } y \geq 0, \alpha>0 .
$$




$$
F(x)=e^{-\frac{\alpha^{2}}{t^{2}}}
$$

If $\mathrm{a}=1$ and $\mathrm{b}=1$, then (1.1) reduces to the two parameter Inverse Weibull distribution(IW), i.e.,

$$
f(y)=\beta \alpha^{-\beta} y^{-(\beta+1)} e^{-(\alpha y)^{-\beta}}, \quad \text { for } y \geq 0, \alpha>0, \beta>0 \text {. }
$$

$$
F(x)=e^{-(\alpha y)^{-\beta}}
$$

\section{The Conclusions}

A new reliability model (Beta Inverse Weibull distribution) was introduced by Khan 2010 as the logit of a beta random variable. This model is based on BIW and it offers more advantages than the IW. The smallest, largest, or middle observations for a give random sample was computed. The basic distribution theory of order statistics (ordered random variables) was developed for both finite and asymptotic random samples. This research considers order statistics as a simple robust location estimator of location generated from Beta inverse Weibull (BIW) distribution. The cumulative distribution and the minimum and maximum density were also presented, the exact expression for the single and double moments of order statistics from BIW distribution have been derived and the doubly truncated BIW was derived. 


\section{References}

Aboutahoun,A.W and Al-Otaibi,N.M.(2009). Recurrence relations between moments of order statistics from doubly truncated Makeham distribution.Computational and applied mathematics Volume28, N.3,pp. 277-290

BalakrishnanN. and Chan P. S.,(1998).Log-gamma order statistics and linear estimation of parameters, In: Balakrishnan, N. and Rao, C. R. (Eds.), Handbook of Statistics, Vol.17. Elsevier Science, Amsterdam, (1998), 6183.

Keller, A. Z. \&Kamath, A. R. R. (1982). Alternative reliability models for mechanical systems. In Proceedings of the Third International Conference on Reliability and Maintainability,pp. 411-415.

Khan,M.S (2010). The Beta inverse Weibull. International Transactions in Mathematical Sciences and Computer. Volume 3, No. 1, 2010, pp. 113-119

Mahmoud M. A. Sultan W., K. S. and Amer S. M.(2003) Order statistics from inverse Weibull distribution and associated inference, Computational Statistics \&DataAnalysis, 42, 149163.

Mohamed.S.A (2013).Bayesian Estimation of the Scale Parameter Under Asymmetric Loss Functions to the Beta Inverse Weibull Distribution. SalehKamel canter for Islamic economic. no 49.437-456 
Order statistics from Beta inverse Weibull distribution

$\sim$ Abeer S.Mohamed

Mohamed.S.A (2014).Comparison Study Between the Maximum Likelihood and Bayesian Estimation for the Beta Inverse Wiebull Distribution

SalehKamel canter for Islamic economic. no 52381-396

RaqabM. Z. and M. Ahsanullah,(2001) Estimation of the location and scale parameters of generalized exponential distribution based on order statistics, J. Statist. Comput. Simul., 69, 109-123

ShawkyA. I. and Bakoban R. A.(2009). Order Statistics from Exponentiated Gamma Distribution and Associated Inference. Int. J. Contemp. Math. Sciences, Vol. 4, no. 2, 71 91 